\title{
Negative Susceptibility Vessel Sign and Underlying Intracranial Atherosclerotic Stenosis in Acute Middle Cerebral Artery Occlusion
}

\author{
S.K. Kim, W. Yoon, T.W. Heo, M.S. Park, and H.K. Kang
}

\begin{abstract}
BACKGROUND AND PURPOSE: The role of MR imaging in predicting underlying intracranial atherosclerotic stenosis before endovascular stroke therapy has not been studied. Our aim was to determine the diagnostic value of the negative susceptibility vessel sign on T2*-weighted gradient-echo MR imaging for predicting underlying intracranial atherosclerotic stenosis in patients with acute MCA occlusion.
\end{abstract}

MATERIALS AND METHODS: Ninety-one consecutive patients with acute stroke because of MCA occlusion underwent gradient-echo MR imaging and MRA before endovascular therapy. The negative susceptibility vessel sign was defined as an absence of a hypointense signal change within the occluded MCA on gradient-echo imaging. Underlying intracranial atherosclerotic stenosis was determined by conventional angiography. The sensitivity, specificity, predictive values, and accuracy of the negative susceptibility vessel sign for predicting the presence of underlying intracranial atherosclerotic stenosis were assessed.

RESULTS: The negative susceptibility vessel sign was identified in 42 (46.1\%) of 91 patients, and 18 (19.8\%) patients had an underlying intracranial atherosclerotic stenosis responsible for acute ischemic symptoms. The negative susceptibility vessel sign was more frequently observed in patients with intracranial atherosclerotic stenosis than in those without it (100\% versus $32.9 \%, P<.001)$. In the prediction of an underlying intracranial atherosclerotic stenosis, the negative susceptibility vessel sign had $100 \%$ sensitivity, $67.1 \%$ specificity, $42.9 \%$ positive predictive value, $100 \%$ negative predictive value, and an accuracy of $73.6 \%$.

CONCLUSIONS: The negative susceptibility vessel sign on gradient-echo MR imaging is a sensitive marker with a high negative predictive value for the presence of an underlying intracranial atherosclerotic stenosis in patients with acute ischemic stroke because of MCA occlusions. The susceptibility vessel sign can be used in decision-making when performing subsequent endovascular revascularization therapy in patients with acute MCA occlusions.

ABBREVIATIONS: GRE = gradient-echo; ICAS = intracranial atherosclerotic stenosis; SBT = stent-based thrombectomy; SVS = susceptibility vessel sign

I ntracranial atherosclerotic stenosis (ICAS) is one of the most common causes of ischemic stroke worldwide and is particularly prevalent in Asian, black, Hispanic, and Indian populations. ${ }^{1,2}$ Underlying ICAS can be a hidden cause of refractory occlusions following modern mechanical thrombectomy procedures, such as stent-based thrombectomy (SBT) or manual aspiration thrombectomy in patients with acute ischemic stroke. ${ }^{3}$ Thus, preprocedural identification of underlying ICAS in patients

Received November 9, 2014; accepted after revision January 14, 2015.

From the Departments of Radiology (S.K.K., W.Y., T.W.H., H.K.K.) and Neurology (M.S.P.), Chonnam National University Medical School, Chonnam National University Hospital, Gwangju, Republic of Korea.

Please address correspondence to Woong Yoon, MD, Department of Radiology, Chonnam National University Hospital, 42 Jebong-Ro, Dong-Gu, Gwangju, 501-757, Republic of Korea; e-mail: radyoon@jnu.ac.kr

http://dx.doi.org/10.3174/ajnr.A4280 with intracranial large-vessel occlusions is of particular importance when performing endovascular stroke therapy.

On MR imaging, hypointense signal changes in the occluded MCA on gradient-echo (GRE) sequences have been described as a susceptibility vessel sign (SVS). ${ }^{4,5}$ The pathophysiologic basis for this sign in patients with an acute MCA occlusion is a T2-shortening effect of the intracellular deoxyhemoglobin component in erythrocytes within the clot. ${ }^{4}$ Several researchers showed that the SVS was more commonly seen in red blood cell-dominant and mixed clots than in fibrin-dominant clots and could therefore reflect the composition of the clots. ${ }^{6}$ In addition, GRE SVS was observed more frequently in patients with cardiogenic embolic stroke than in those with other stroke subtypes. ${ }^{5}$ In contrast, the absence of the GRE SVS might be associated with a smaller clot burden or a fibrin-dominant clot that forms as a result of rupture of the underlying atherosclerotic plaque. Thus, we hypothesized 
that the absence of the GRE SVS despite the presence of intracranial large-vessel occlusions could indicate an underlying ICAS in patients with acute ischemic stroke within 6 hours of stroke onset, and we termed this finding the "negative SVS." Despite the widespread use of MR imaging for the diagnosis of acute ischemic stroke, the role of MR imaging in predicting underlying ICAS before endovascular therapy has not been studied, to our knowledge. In this study, we evaluated the diagnostic value of the negative SVS on GRE imaging for the prediction of underlying ICAS in patients with acute MCA occlusion.

\section{MATERIALS AND METHODS Patients}

From January 2011 to February 2013, 101 consecutive patients presenting with acute ischemic stroke from MCA occlusion within 6 hours of symptom onset were treated with multimodal endovascular revascularization therapy at a tertiary university hospital. Of these patients, those who did not undergo the GRE sequence $(n=8)$ or had GRE images of insufficient quality for reliable evaluation because of motion artifacts $(n=2)$ were excluded, leaving 91 patients in this study. This retrospective study analyzed the radiologic and clinical data collected prospectively from these 91 patients. The institutional ethics committee approved this retrospective analysis and waived the requirements for informed consent on the basis of the study design.

\section{MR Imaging Analysis}

MR imaging examinations were performed by using a $1.5 \mathrm{~T}$ unit (Signa HDxt; GE Healthcare, Milwaukee, Wisconsin). Before the endovascular procedure, patients underwent MR imaging, including DWI, GRE, FLAIR, 3D TOF MRA, and perfusion imaging. GRE sequence parameters included a TR of $750-800 \mathrm{~ms}$, TE of $14 \mathrm{~ms}$, flip angle of $20^{\circ}$, section thickness of $4 \mathrm{~mm}$, intersection gap of $0 \mathrm{~mm}$, and FOV of $230 \times 230 \mathrm{~mm}$. 3D TOF MRA parameters included a TR of $24 \mathrm{~ms}$, TE of $6.9 \mathrm{~ms}$, flip angle of $20^{\circ}$, and FOV of $240 \times 240 \mathrm{~mm}$.

All MR images were retrospectively reviewed by 2 neuroradiologists (with 1 and 3 years of experience, respectively) who were blinded to the findings from conventional angiography. Conclusions were reached by consensus regarding the presence or absence of the negative SVS on GRE imaging. A negative SVS was defined as the absence of the GRE SVS in the occluded MCA. GRE SVS was defined as a hypointense signal change on GRE imaging within the occluded MCA, in which the diameter of the hypointense signal exceeds that of the contralateral MCA., ${ }^{4,5}$

\section{Catheter Angiography and Endovascular Therapy}

On admission, a stroke neurologist performed an assessment based on the NIHSS. The inclusion criteria for endovascular therapy were as follows: presentation within 6 hours of stroke onset for an anterior circulation stroke; baseline NIHSS score of $\geq 4$; no intracranial hemorrhage detected on the cranial CT or MR imaging; no flow-related enhancement in the M1 or M2 segment of MCA on the MRA; a target mismatch pattern on multimodal MR imaging based on visual estimation (time-to-peak map of perfusion imaging showing a lesion volume $\geq 30 \%$ larger than that detected with DWI); infarct volume on DWI less than one-third of the MCA territory; and a premorbid mRS score of $\leq 3$.

Underlying ICAS was determined by conventional angiography when the severe stenosis ( $\geq 70 \%$ ) of the MCA, measured by the Warfarin-Aspirin Symptomatic Intracranial Disease criteria, ${ }^{7}$ was seen on the initial diagnostic angiography or on the follow-up angiography after the first-line SBT. For underlying ICAS that was seen after the first-line SBT, ICAS was determined if the stenotic lesion remained unchanged on the follow-up angiography performed $>5$ minutes after intra-arterial injection of a vasodilator (50 $\mu \mathrm{g}$ of nitroglycerin) through the guide catheter. Angiographic images were retrospectively reviewed by a neuroradiologist (with 8 years of experience) who was blinded to the findings from MR imaging.

All conventional angiography and endovascular therapy were performed by a single interventional neuroradiologist with 12 years of experience in neurovascular intervention. For each patient, written informed consent for angiography and endovascular therapy was obtained from a family member. Cerebral angiography and endovascular therapy were performed with the patient under conscious sedation. In cases of agitation, an intravenous bolus of midazolam was given and repeated if necessary. When the initial diagnostic angiography showed arterial occlusion, SBT with a Solitaire stent (Covidien, Irvine, California) was performed as the first-line endovascular treatment. When SBT was unsuccessful, additional mechanical approaches were performed, including manual aspiration thrombectomy with a Penumbra System perfusion catheter (Penumbra, Alameda, California) and intra-arterial urokinase infusion with or without clot disruption with a microguidewire. The details of the techniques used for SBT, manual aspiration thrombectomy, and clot disruption with a microwire were described previously. ${ }^{8,9}$

All patients with underlying ICAS were treated with intracranial angioplasty with or without stent placement. Intracranial angioplasty was performed with a Gateway PTA balloon catheter (Stryker, Kalamazoo, Michigan). The diameter of the balloon was undersized to $80 \%$ of the normal vessel diameter just distal to the stenosis, and the shortest length was used to cover the lesion. The balloon was inflated slowly 1 or 2 times by using a screw-type pressure inflation device at $4-6$ atm for 30-60 seconds. Whenever possible, intracranial stent placement with the Wingspan stent system (manufactured by Stryker, Kalamazoo, Michigan, for Boston Scientific, Natick, Massachusetts) was performed after intracranial angioplasty. The stent diameter was sized to exceed the diameter of the normal vessel by $0.5 \mathrm{~mm}$. The stent length was selected to cover the entire stenotic segment. Patients who underwent intracranial angioplasty with or without stent placement received aspirin and clopidogrel bisulfate (Plavix) orally via a nasogastric tube immediately after the procedure, and dual antiplatelet therapy was continued for at least 3 months after the procedure. Revascularization status was assessed on the final angiogram and was classified according to the modified TICI scale, ${ }^{10}$ and successful revascularization was defined as a modified TICI grade $2 \mathrm{~b}$ or 3 . Clinical outcome was assessed by a stroke neurologist by using the mRS during an outpatient visit 3 months after treatment. If patients were unable to attend the outpatient clinic, outcomes were obtained via telephone interview. A good 
clinical outcome was defined as an mRS score of $\leq 2$. Stroke subtypes were determined by a stroke neurologist according to the classification of the Trial of Org 10172 in Acute Stroke Treatment. $^{11}$

\section{Statistical Analysis}

First, we compared differences between the patients with a negative SVS (negative SVS group) and those with a GRE SVS (positive SVS group) and then differences between the ICAS group (patients with underlying ICAS) and the non-ICAS group (patients without underlying ICAS) separately. Differences in baseline characteristics and clinical outcomes between the 2 groups were compared by using bivariate analysis. The Pearson $\chi^{2}$ or Fisher exact test was used for categoric variables, and the Mann-Whitney $U$ test, for continuous variables. Next, diagnostic performance parameters, including sensitivity, specificity, positive predictive value, negative predictive value, and the diagnostic accuracy of the negative SVS for the prediction of underlying ICAS, were calculated. Finally, independent associations of clinical outcomes with other clinical and radiologic factors were determined with a multivariate logistic regression analysis. The variables tested in the logistic regression models were those with $P<.2$ in the univariate analysis. All statistical analyses were performed with SPSS software (Version 20.0; IBM, Armonk, New York). $P<.05$ was a statistically significant difference.

\section{RESULTS}

Of the 91 patients ( 52 men and 39 women; age range, 40-92 years) with acute MCA occlusions treated with multimodal endovascular therapy, a negative SVS on GRE imaging was identified in 42 (46.1\%) patients. On conventional angiography, 18 (19.8\%) of 91 patients had an underlying ICAS in the MCA, which was responsible for their acute ischemic symptoms. Of 18 patients with underlying ICAS, 8 exhibited a severe $(\geq 70 \%)$ stenosis of the target artery on the initial diagnostic conventional angiography despite the 3D TOF MRA demonstrating an absence of flow-related enhancement in the M1 and M2 segments of the MCA. The remaining 10 patients had an occlusion visible on the initial conventional angiography and had a severe stenosis at the previous occlusion site after SBT. Of these 10 patients, only 3 had visible thrombi that were captured with a Solitaire stent. The remaining 7 patients had no captured thrombi despite several passages of the Solitaire stent. Thus, overall, 15 of 18 patients (83\%) with underlying ICAS had no apparent clot burden in our study. The occlusion sites were in the M1 segment in all patients with underlying ICAS, while those in the non-ICAS group were in the M1 $(n=66)$ and M2 $(n=7)$ segments.

\section{Negative SVS versus Positive SVS}

The results of comparisons between the patients with a negative SVS and those with a positive SVS are shown in Table 1. Diabetes and dyslipidemia were more frequent in patients in the negative SVS group $(P=.046$ and $P=.011)$, whereas atrial fibrillation was more frequent in the positive SVS group $(P=.011)$. The mean baseline NIHSS score was significantly lower in the negative SVS group compared with the positive SVS group (9.5 versus 13, $P=$ .003). For stroke subtypes, the negative SVS was significantly as-
Table 1: Comparison between the negative SVS group and positive SVS group

\begin{tabular}{|c|c|c|c|}
\hline & $\begin{array}{l}\text { Negative SVS } \\
\text { Group }(n=42)\end{array}$ & $\begin{array}{l}\text { Positive SVS } \\
\text { Group }(n=49)\end{array}$ & $\begin{array}{c}P \\
\text { Value }\end{array}$ \\
\hline Age (yr) & $69.1 \pm 11.60$ & $68.4 \pm 11.40$ & NS \\
\hline Male sex & $21(50 \%)$ & $31(63.3 \%)$ & NS \\
\hline \multicolumn{4}{|l|}{ Risk factors } \\
\hline Hypertension & $26(61.9 \%)$ & $27(55.1 \%)$ & NS \\
\hline Diabetes mellitus & $10(23.8 \%)$ & $4(8.2 \%)$ & .046 \\
\hline Coronary artery disease & $3(7.1 \%)$ & $2(4.1 \%)$ & NS \\
\hline Dyslipidemia & $19(45.2 \%)$ & $10(20.4 \%)$ & .011 \\
\hline Smoking & $8(19.0 \%)$ & $17(34.7 \%)$ & NS \\
\hline Atrial fibrillation & $12(28.6 \%)$ & $27(55.1 \%)$ & .011 \\
\hline Congestive heart failure & $1(2.4 \%)$ & $1(2.0 \%)$ & NS \\
\hline History of stroke or TIA & $7(16.7 \%)$ & $5(10.2 \%)$ & NS \\
\hline \multicolumn{4}{|l|}{ Occlusion sites } \\
\hline Ml segment & $38(90.5 \%)$ & $46(93.9 \%)$ & NS \\
\hline M2 segment & $4(9.5 \%)$ & $3(6.1 \%)$ & NS \\
\hline IV thrombolysis & $20(47.6 \%)$ & $34(69.4 \%)$ & NS \\
\hline Time to procedure (min) & $240 \pm 115.9$ & $250 \pm 74.3$ & NS \\
\hline Procedure time (min) & $31.5 \pm 9.3$ & $30 \pm 18.0$ & NS \\
\hline Time to revascularization (min) & $280 \pm 117.8$ & $276 \pm 78.3$ & NS \\
\hline Baseline NIHSS score & $9.5 \pm 3.7$ & $13 \pm 3.7$ & .003 \\
\hline \multicolumn{4}{|l|}{ Stroke subtypes } \\
\hline Cardioembolic & $11(26.2 \%)$ & $29(59.2 \%)$ & .002 \\
\hline Large artery atherosclerosis & $24(57.1 \%)$ & $9(18.4 \%)$ & $<.001$ \\
\hline Others & $0 \%$ & $0 \%$ & NS \\
\hline Undetermined & $7(16.7 \%)$ & $11(22.4 \%)$ & NS \\
\hline $\mathrm{m}-\mathrm{TICl} 2 \mathrm{~b}$ or 3 & $38(90.5 \%)$ & $39(79.6 \%)$ & NS \\
\hline mRS 0-2 & $28(66.7 \%)$ & $21(42.9 \%)$ & .023 \\
\hline Mortality & $5(11.9 \%)$ & $4(8.2 \%)$ & NS \\
\hline
\end{tabular}

Note:- $\mathrm{m}$ - $\mathrm{TICl}$ indicates modified TICl; NS, non-significant.

sociated with large-artery atherosclerosis. Of the 33 patients with large-artery atherosclerosis, $24(72.7 \%)$ had a negative SVS (Fig $1)$, whereas $9(27.3 \%)$ had a positive SVS $(P<.001)$. In contrast, a positive SVS was significantly associated with cardioembolic stroke: Twenty-nine $(72.5 \%)$ of 40 patients with cardioembolic stroke had a positive SVS (Fig 2) and 11 (27.5\%) had a negative SVS $(P=.002)$. Good outcomes occurred significantly more frequently in the negative SVS group than in the positive SVS group (66.7\% versus $42.9 \%, P=.023)$.

\section{ICAS versus Non-ICAS}

The results of comparison between the ICAS group and the nonICAS group are shown in Table 2. Patients in the ICAS group were younger than those in the non-ICAS group $(P=.026)$. Diabetes and dyslipidemia were more frequent in patients with ICAS $(P=$ .002 and $P<.001$, respectively), whereas atrial fibrillation was more frequent in the non-ICAS group $(P<.001)$. The mean baseline NIHSS score was significantly lower in the ICAS group compared with the non-ICAS group (9.8 versus $12.7, P=.003$ ). There were no significant differences between the 2 groups in other risk factors, arterial occlusion sites, use of IV thrombolysis, procedure time, and time to revascularization.

All patients with ICAS had a negative SVS, whereas only 24 patients in the non-ICAS group had a negative SVS (100\% versus $32.9 \%, P<.001)$. The incidence of negative SVS according to occlusion site is summarized in Table 3 . In the prediction of an underlying ICAS, a negative SVS had 100\% (95\% CI, 90\%-100\%) sensitivity, 67.1\% (95\% CI, 55\%-77\%) specificity, 42.9\% (95\% CI, $28 \%-59 \%$ ) positive predictive value, $100 \%$ (95\% CI, $90 \%-$ $100 \%$ ) negative predictive value, and $73.6 \%$ accuracy.

All 18 patients with underlying ICAS received intracranial an- 

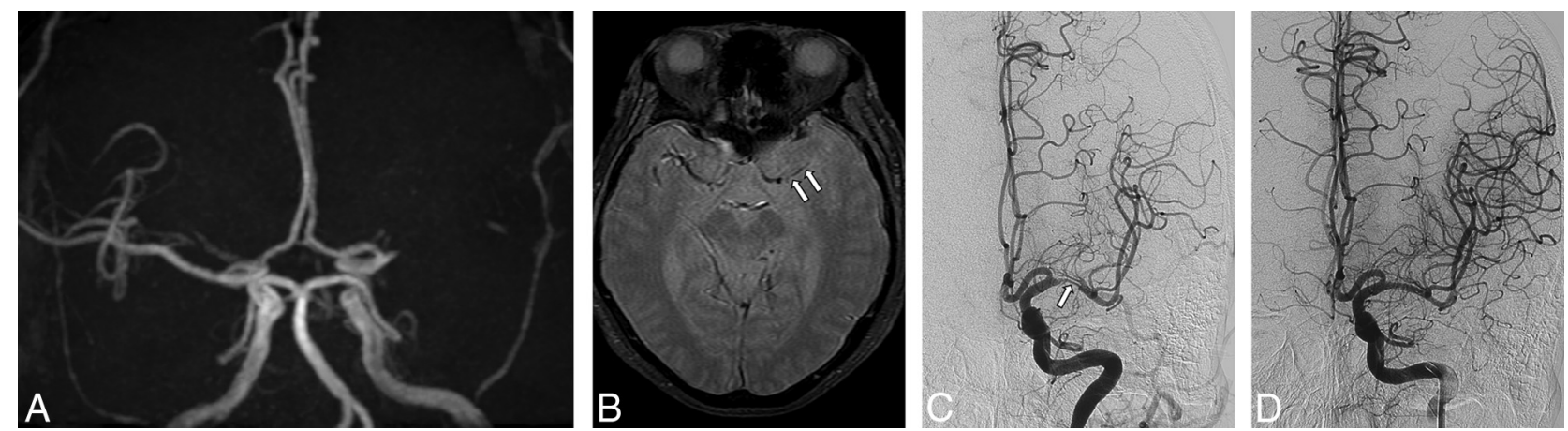

FIG 1. Brain images from a 74-year-old man with acute ischemic stroke and atherosclerotic stenosis in the MCA. A, 3D TOF MR angiography shows the occlusion in the proximal M1 segment of the left MCA. B, Axial gradient-echo image reveals a negative susceptibility vessel sign (arrows) in the M1 segment of the left MCA. C, Angiography after 1 passage of the Solitaire stent identifies a severe stenosis (arrow) in the proximal $\mathrm{Ml}$ segment of the left MCA. No thrombi were retrieved with the Solitaire stent. $D$, Angiography after intracranial angioplasty with stent placement shows complete revascularization in the left MCA territory.
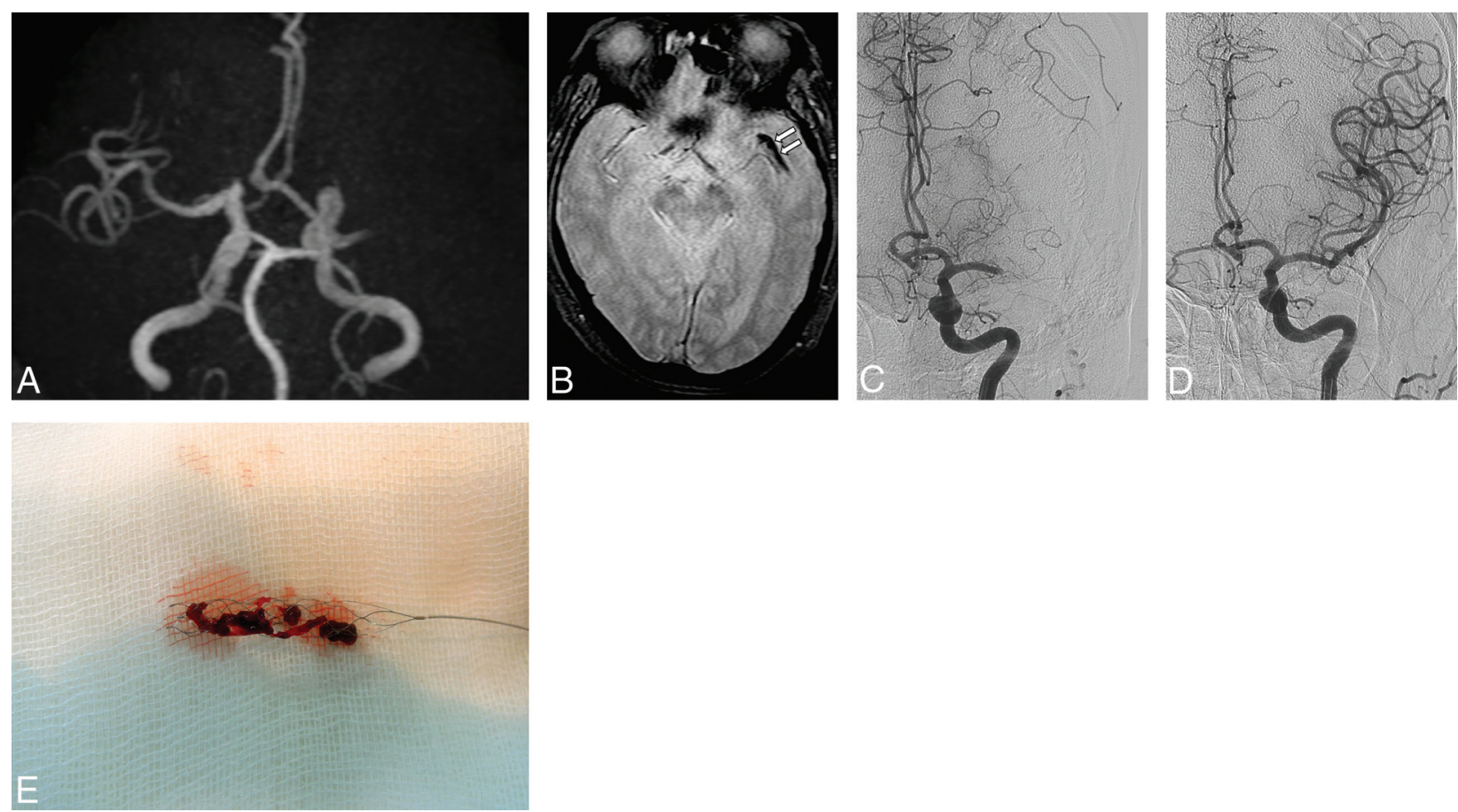

FIG 2. Brain images from a 74-year-old man with acute ischemic stroke and atrial fibrillation. A, 3D TOF MR angiography shows the occlusion in the M1 segment of the left MCA. B, Axial gradient-echo image reveals a positive susceptibility vessel sign (arrows) in the distal M1 segment of the left MCA. C, Conventional angiography shows the occlusion in the M1 segment of the left MCA. D, Angiography after 1 passage of the Solitaire stent shows complete revascularization in the left MCA territory. E, Photograph demonstrates red clots retrieved with a Solitaire stent.

gioplasty with or without stent placement. Overall, successful revascularization (modified TICI $\geq 2 \mathrm{~b}$ ) was achieved in $84.6 \%$ (77/ 91) of patients. Successful revascularization was achieved more frequently in the ICAS group than in the non-ICAS group $(100 \%$ versus $80.8 \%, P=.043$ ). Good outcomes occurred significantly more frequently in the ICAS group than in the non-ICAS group (77.8\% versus $47.9 \%, P=.034)$. There was no newly developed arterial occlusion distal to the angioplasty site on postangioplasty angiograms in all patients who underwent intracranial angioplasty with or without stent placement.

\section{Predictors of Clinical Outcome}

Multivariate analysis showed that age (OR, 1.067; 95\% CI, 1.013$1.124 ; P=.014)$ and revascularization $(\mathrm{OR}, 9.180$; 95\% CI, 1.545-
54.526; $P=.015)$ were independent predictors of good clinical outcome when adjusting for age, sex, dyslipidemia, atrial fibrillation, coronary artery disease, IV thrombolysis, baseline NIHSS score, revascularization (modified TICI $\geq 2 b$ ), posttreatment intracranial hemorrhage, ICAS, and SVS.

\section{DISCUSSION}

In the present study, a negative SVS on GRE imaging was a highly sensitive marker with a high negative predictive value for the presence of an underlying ICAS in the setting of acute stroke because of an acute MCA occlusion. A negative SVS on GRE imaging was significantly associated with an underlying ICAS in the occluded MCA (100\% in the ICAS group versus $32.9 \%$ in the non-ICAS 
Table 2: Comparison between the ICAS group and non-ICAS group

\begin{tabular}{|c|c|c|c|}
\hline & $\begin{array}{l}\text { ICAS } \\
\text { Group } \\
(n=18)\end{array}$ & $\begin{array}{c}\text { Non-ICAS } \\
\text { Group } \\
(n=73)\end{array}$ & $P$ Value \\
\hline Age (yr) & $63.8 \pm 10.4$ & $69.9 \pm 11.4$ & .026 \\
\hline Male sex & $11(61.1 \%)$ & $41(56.2 \%)$ & NS \\
\hline \multicolumn{4}{|l|}{ Risk factors } \\
\hline Hypertension & $12(66.7 \%)$ & $41(56.2 \%)$ & NS \\
\hline Diabetes mellitus & $7(38.9 \%)$ & $7(9.6 \%)$ & .002 \\
\hline Coronary artery disease & $0(0 \%)$ & $5(6.8 \%)$ & NS \\
\hline Dyslipidemia & $12(66.7 \%)$ & $17(23.2 \%)$ & $<.001$ \\
\hline Smoking & $3(16.7 \%)$ & $22(30.1 \%)$ & NS \\
\hline Atrial fibrillation & $1(5.6 \%)$ & $38(52.1 \%)$ & $<.001$ \\
\hline Congestive heart failure & $0(0 \%)$ & $2(2.7 \%)$ & NS \\
\hline History of stroke or TIA & $1(5.6 \%)$ & $11(15.1 \%)$ & NS \\
\hline \multicolumn{4}{|l|}{ Occlusion sites } \\
\hline M1 segment & $18(100 \%)$ & $66(90.4 \%)$ & NS \\
\hline M2 segment & $0(0 \%)$ & $7(9.6 \%)$ & NS \\
\hline IV thrombolysis & $7(38.9 \%)$ & $47(64.4 \%)$ & NS \\
\hline Time to procedure (min) & $253.8 \pm 115.9$ & $250.5 \pm 74.2$ & NS \\
\hline Procedure time (min) & $31.9 \pm 9.3$ & $33.6 \pm 18.0$ & NS \\
\hline Time to revascularization (min) & $285.8 \pm 117.8$ & $284.3 \pm 78.2$ & NS \\
\hline Baseline NIHSS score & $9.8 \pm 3.6$ & $12.7 \pm 3.7$ & .003 \\
\hline $\mathrm{m}-\mathrm{TICl} 2 \mathrm{~b}$ or 3 & $18(100 \%)$ & $59(80.8 \%)$ & .043 \\
\hline mRS 0-2 & 14 (77.8\%) & 35 (47.9\%) & .034 \\
\hline Mortality & $1(5.6 \%)$ & $8(10.9 \%)$ & NS \\
\hline N-SVS & 18 (100\%) & $24(32.9 \%)$ & $<.001$ \\
\hline
\end{tabular}

Note:- N-SVS indicates negative susceptibility vessel sign; m-TICl, modified TICl; NS, non-significant.

Table 3: Summary of GRE MRI findings according to occlusion sites

\begin{tabular}{|c|c|c|c|c|}
\hline \multirow[b]{2}{*}{ Location } & \multicolumn{2}{|c|}{$\begin{array}{l}\text { ICAS Group } \\
(n=18)\end{array}$} & \multicolumn{2}{|c|}{$\begin{array}{c}\text { Non-ICAS Group } \\
(n=73)\end{array}$} \\
\hline & $\begin{array}{c}\text { SVS } \\
\text { Absent }\end{array}$ & $\begin{array}{c}\text { SVS } \\
\text { Present }\end{array}$ & $\begin{array}{c}\text { SVS } \\
\text { Absent }\end{array}$ & $\begin{array}{l}\text { SVS } \\
\text { Present }\end{array}$ \\
\hline M1 & 18 & 0 & 20 & 46 \\
\hline$M 2$ & 0 & 0 & 4 & 3 \\
\hline Total $(n=91)$ & 18 & 0 & 24 & 49 \\
\hline
\end{tabular}

group, $P<.001)$ with a high sensitivity $(100 \%)$ and a high negative predictive value (100\%). For stroke subtypes, 72.7\% (24/33) of patients with large-artery atherosclerosis had a negative SVS and $72.5 \%(29 / 40)$ of patients with cardioembolic stroke had a positive SVS in our study. In comparison, Cho et $\mathrm{al}^{5}$ reported that $83.7 \%$ of those with large-artery atherosclerosis had a negative SVS and $77.5 \%$ of those with cardioembolic stroke had a positive SVS.

Several studies reported that GRE MR imaging can be used to depict blood clots within occluded intracranial arteries and to evaluate the clot composition in the setting of acute ischemic stroke. ${ }^{4,6,12}$ Paramagnetic intracellular deoxyhemoglobin is present at high concentrations in acute clots, leading to a nonuniform magnetic field that causes marked signal loss on the GRE sequence because of dephasing of spins. ${ }^{13}$ Recently, several investigators reported the results of histologic studies analyzing the composition of the clots retrieved by mechanical thrombectomy in patients with acute ischemic stroke. ${ }^{6,14-16}$ These studies demonstrated that the presence of an SVS on GRE imaging was notably associated with a higher red blood cell content in the clots, whereas an absence of SVS was associated with fibrin-rich clots. Fibrin-dominant clots are usually formed because of injury to the endothelium overlying the complicated atheromatous plaque. Clots formed at the site of underlying ICAS mainly consist of a fibrin network superimposed on the underlying platelet aggregates $^{5,17}$ and would not appear as a hypointense vessel on GRE imaging because of the lack of deoxyhemoglobin. The results from our study support this hypothesis; all patients with underlying ICAS in the occluded MCA had a negative SVS on GRE imaging.

Another plausible explanation for the high incidence of negative SVSs in patients with underlying ICAS would be that such patients had a smaller clot burden compared with those having other stroke subtypes. In our study, 8 of 18 patients with underlying ICAS had a severe stenosis on the initial conventional angiography. Of the 10 patients who underwent SBT with the Solitaire stent because of an occlusion seen on the initial conventional angiography, only 3 patients had visible thrombi that were captured with a stent. The remaining 7 patients had no captured thrombi despite several passages of the stent and had a severe stenosis at the previous occlusion site without angiographic evidence of distal migration of thrombi on follow-up angiography. Thus, overall, 15 of 18 patients (83\%) with underlying ICAS had no apparent clot burden in our study.

In our study, the negative SVS showed low specificity and low positive predictive values for predicting ICAS because of a high incidence of false-positive findings (24 cases) in the non-ICAS group. The false-positive cases showing a negative SVS despite the absence of ICAS in the non-ICAS group might be attributed to the size or aging of the clots and their components. Red blood celldominant clots that are smaller than the section thickness of the GRE sequence may not demonstrate GRE SVS. In addition, fresh clots cannot demonstrate hypointense signal changes on GRE MR imaging because the main component of hyperacute clots would be oxyhemoglobin. ${ }^{18}$

ICAS also can be detected and evaluated with high-resolution vessel wall MR imaging. Recent advances in MR imaging have made it possible to reliably image intracranial vessel wall pathologies, including ICAS. ${ }^{19}$ Vessel wall MR imaging can reveal the presence of atherosclerotic plaques or focal thickening and enhancement of the intracranial vessel wall in patients with ICAS. In addition, recent studies showed that vessel wall MR imaging might be used for the characterization of intracranial atherosclerotic plaques to assess the vulnerability of these plaques. ${ }^{20} \mathrm{~A}$ highresolution vessel wall $\mathrm{MR}$ imaging sequence may be included in the clinical scan protocol for ischemic stroke in the future. However, it is generally not possible to obtain vessel wall images of sufficient quality for interpretation from patients with stroke in the hyperacute period because of long scan times.

Although the optimal treatment for underlying ICAS in patients presenting with hyperacute stroke remains unknown, ${ }^{21}$ the identification of underlying ICAS before endovascular therapy could help interventionists make appropriate therapeutic decisions. ${ }^{5,18,22}$ Recently, Kang et al ${ }^{22}$ reported that patients with underlying ICAS who presented with acute stroke had an increased tendency for instant reocclusion of the treated arteries after the initial mechanical thrombectomy. They suggested that the infusion of intra-arterial antiplatelet agents in combination with a thrombolytic agent might reduce the rate of reocclusion in pa- 
tients with acute stroke with underlying ICAS. In our study, we performed intracranial angioplasty, with or without stent placement, in all patients with underlying ICAS, which resulted in high rates of revascularization (100\%) and good outcome $(77.8 \%)$ and low mortality rates $(5.6 \%)$. Although a randomized controlled trial (Stenting and Aggressive Medical Management for Preventing Recurrent stroke in Intracranial Stenosis) failed to show a benefit for intracranial stent placement over intensive medical management in preventing stroke recurrence in high-risk patients with symptomatic ICAS, ${ }^{23}$ our study suggests that intracranial angioplasty, with or without stent placement, remains a promising method in the setting of hyperacute stroke secondary to underlying ICAS.

This single-center retrospective study has limitations inherent to this type of case series, including the lack of a prospective study design. In our study, the section thickness of the GRE sequence was $4 \mathrm{~mm}$. Red blood cell-dominant clots smaller than $4 \mathrm{~mm}$ may not be seen on GRE images; this possibility might have affected the results of the study. In addition, it might be difficult to differentiate underlying stenosis from residual emboli after mechanical thrombectomy on angiograms. However, there was no newly developed arterial occlusion distal to the angioplasty site when comparing pre- and postangioplasty angiograms in all patients, which could confirm stenotic lesions not being caused by residual emboli. Finally, this study was conducted in an Asian country in which ICAS is more prevalent than in Europe and the United States. Thus, a high incidence $(19.8 \%)$ of patients with ICAS received endovascular therapy in the setting of hyperacute stroke. However, the global burden of ICAS is increasing, and it is generally accepted that ICAS is now a major worldwide stroke problem that cannot be ignored. ${ }^{21}$

\section{CONCLUSIONS}

This study suggests that a negative SVS on GRE MR imaging is a sensitive marker with a high negative predictive value for the presence of an underlying ICAS in patients with acute ischemic stroke because of MCA occlusion. A predictor such as this would be helpful for interventionists when performing subsequent endovascular stroke therapy.

\section{REFERENCES}

1. Holmstedt CA, Turan TN, Chimowitz MI. Atherosclerotic intracranial arterial stenosis: risk factors, diagnosis, and treatment. Lancet Neurol 2013;12:1106-14

2. Qureshi AI, Caplan LR. Intracranial atherosclerosis. Lancet 2014; 383:984-98

3. Shi ZS, Liebeskind DS, Loh Y, et al. Predictors of subarachnoid hemorrhage in acute ischemic stroke with endovascular therapy. Stroke 2010;41:2775-81

4. Rovira A, Orellana P, Alvarez-Sabin J, et al. Hyperacute ischemic stroke: middle cerebral artery susceptibility sign at echo-planar gradient-echo MR imaging. Radiology 2004;232:466-73

5. Cho KH, Kim JS, Kwon SU, et al. Significance of susceptibility vessel sign on $\mathrm{T} 2{ }^{\star}$-weighted gradient echo imaging for identification of stroke subtypes. Stroke 2005;36:2379-83

6. Liebeskind DS, Sanossian N, Yong WH, et al. CT and MRI early vessel signs reflect clot composition in acute stroke. Stroke 2011; 42:1237-43

7. Samuels OB, Joseph GJ, Lynn MJ, et al. A standardized method for measuring intracranial arterial stenosis. AJNR Am J Neuroradiol 2000;21:643-46

8. Yoon W, Jung MY, Jung SH, et al. Subarachnoid hemorrhage in a multimodal approach heavily weighted toward mechanical thrombectomy with Solitaire stent in acute stroke. Stroke 2013;44:414-19

9. Kim SK, Yoon W, Moon SM, et al. Outcomes of manual aspiration thrombectomy for acute ischemic stroke refractory to stent-based thrombectomy. J Neurointerv Surg 2015;7:473-77

10. Zaidat OO, Yoo AJ, Khatri P, et al. Recommendations on angiographic revascularization grading standards for acute ischemic stroke: a consensus statement. Stroke 2013;44:2650-63

11. Adams HP Jr, Bendixen BH, Kappelle LJ, et al. Classification of subtype of acute ischemic stroke: definitions for use in a multicenter clinical trial-TOAST. Trial of Org 10172 in Acute Stroke Treatment. Stroke 1993;24:35-41

12. Naggara O, Raymond J, Domingo Ayllon M, et al. T2* "susceptibility vessel sign" demonstrates clot location and length in acute ischemic stroke. PLoS One 2013;8:e76727

13. Patel MR, Edelman RR, Warach S. Detection of hyperacute primary intraparenchymal hemorrhage by magnetic resonance imaging. Stroke 1996;27:2321-24

14. Marder VJ, Chute DJ, Starkman S, et al. Analysis of thrombi retrieved from cerebral arteries of patients with acute ischemic stroke. Stroke 2006;37:2086-93

15. Yuki I, Kan I, Vinters HV, et al. The impact of thromboemboli histology on the performance of a mechanical thrombectomy device. AJNR Am J Neuroradiol 2012;33:643-48

16. Niesten JM, van der Schaaf IC, van Dam L, et al. Histopathologic composition of cerebral thrombi of acute stroke patients is correlated with stroke subtype and thrombus attenuation. PLoS One 2014;9:e88882

17. Brogan GX Jr. Bench to bedside: pathophysiology of acute coronary syndromes and implications for therapy. Acad Emerg Med 2002; 9:1029-44

18. Kimura K, Iguchi Y, Shibazaki K, et al. M1 susceptibility vessel sign on $\mathrm{T}^{*}$ as a strong predictor for no early recanalization after IVt-PA in acute ischemic stroke. Stroke 2009;40:3130-32

19. Dieleman N, van der Kolk AG, Zwanenburg JJ, et al. Imaging intracranial vessel wall pathology with magnetic resonance imaging: current prospects and future directions. Circulation 2014;130: 192-201

20. van der Kolk AG, Zwanenburg JJ, Denswil NP, et al. Imaging the intracranial atherosclerotic vessel wall using 7T MRI: initial comparison with histopathology. AJNR Am J Neuroradiol 2015;36: $694-701$

21. Gorelick PB, Wong KS, Bae HJ, et al. Large artery intracranial occlusive disease: a large worldwide burden but a relatively neglected frontier. Stroke 2008;39:2396-99

22. Kang DH, Kim YW, Hwang YH, et al. Instant reocclusion following mechanical thrombectomy of in situ thromboocclusion and the role of low-dose intra-arterial tirofiban. Cerebrovasc Dis 2014;37: 350-55

23. Derdeyn CP, Chimowitz MI, Lynn MJ, et al. Aggressive medical treatment with or without stenting in high-risk patients with intracranial artery stenosis (SAMMPRIS): the final results of a randomised trial. Lancet 2014;383:333-41 